\title{
Numerical Simulation of Fretting Fatigue for Endless Rope Winch Drum
}

\author{
Nguyen Van Xo \\ Department of Machinery and eguipment industry \\ Ha Noi University of Mining and Geology \\ Ha Noi, Vietnam \\ E-mail address: nguyenvanxo@humg.edu.vn
}

\author{
Golikov Nikolay Sergeevich \\ Department of Mechanical engineering \\ Saint-Petersburg Mining University \\ Saint-Petersburg, Russia \\ E-mail address: golikov_ns@pers.spmi.ru
}

\begin{abstract}
Surface tensile stress and internal shear stress are main sources of the fretting fatigue for endless rope winch drum during operation. To probe the distributions and impact factors, the basic theories of microtribology and contact mechanics are applied to derive analytical expressions. Then, numerical simulation is conducted to discuss effects of the friction coefficient, surrounding angle and coefficient of cylinder generatrix. The results indicate that the maximum surface tensile stress increases with the friction coefficient. The influence of surrounding angle is greater. As the coefficient of cylinder generatrix increases close to 1 , the increasing trend of maximum surface tensile stress becomes gentle. AS for the maximum internal shear stress, the friction coefficient has little effect. Research results have important theoretical significance for rational design and scientific maintenance of the endless rope winch drum.
\end{abstract}

Keywords - endless rope winch, drum, fretting fatigue, numerical simulation

\section{INTRODUCTION}

Improving the reliability of mining hoisting and handling machines can go in different directions. On one side it is the introduction of new methods of processing machine's details [1], on the other side it is a new methods of non-destructive testing in the diagnosis of the technical condition of the elements of mining equipment [2]. However, since about $70 \%$ of the reliability of machines is laid at the stage of their design, a much more significant direction of reliability improvement can be considered mathematical modeling of strength characteristics, fatigue processes and stress state of mining equipment parts.

Endless rope winch is mainly used for material, equipment and personnel transportation in work face of mining area [3]. As the main bearing part, the drum is wrapped by wire rope to provide friction force for power transmission. Fretting fatigue damage is inevitable on the drum in service due to effects of tensile and shear stresses, thus contributing to premature formation of crack and greatly reducing service life of the drum. As a consequence, study on the root and impact factors of fretting fatigue is critical to the normal operation of the whole system.

Fretting refers to the slight movement between two contact surfaces (typically microns), according to different sources, it can be divided into fretting wear and fretting fatigue [4]. Fretting fatigue can accelerate crack initiation and growth, lead to premature destruction of mechanical components. At present, experts and scholars had conducted a deeply research on fretting fatigue mechanism and crack propagation. Eden, Roses and Cunningham [5] experimentally observed for the first time that there was a certain relationship between fretting and fatigue. Mindlin [6] divided contact area into slip region and non-slip region, and computed the stress distribution on contact interface. Zhou [7] proposed micro graphic theory to reveal the operation and injury law of fretting. Hills and Nowell $[8,9]$ enriched and developed the Mindlin theory in the field of mechanical calculation, especially conducted extensive research on the initiation and extension of fatigue crack. However, there are indeed few papers concerned with the mechanical causes of fretting fatigue up to present, further studies are still necessary, practically it has been blank on fretting fatigue research for endless rope winch drum.

In normal conditions, there exists compound effect of normal pressure and tangential force in contact region during the transmission process, The surface tensile stress and internal shear stress are the major sources of fretting fatigue crack, through numerical simulation, study on the impacts of friction coefficient, the surrounding angle and coefficient of cylinder generatrix on maximum surface tensile stress, simultaneous analysis of the effect of friction coefficient on maximum internal shear stress, research results could provide theoretical guidance for fatigue life analysis, furthermore rational design and scientific maintenance of the drum would be available.

\section{CONTACT MODEL ANALYSIS}

For the realization of dual directional traction, the drum is usually designed as conical (see Fig.1.), when it rotates forward, warps one half of the drum, if reverse, wraps the other half, friction drive formula is given in literature [10]

$$
S_{1}=S_{0} e^{\mu \alpha \cos \theta}
$$

where, $S_{1}, S_{0}$ are the rope tensions on heavy and light load side respectively, $e$ is the natural logarithm, $\mu$ is the friction coefficient, $\alpha$ is the surrounding angle, $\theta$ is the inclination angle of cylinder generatrix. 


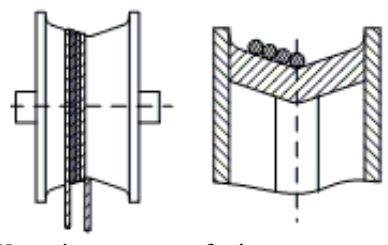

Fig. 1. Wrapping pattern of wire rope onto the drum.

Discrete treatment of wire rope, consider it as be composed of many consecutive cylindrical micro-segments, as shown in Fig. 2, $D$ represents the drum diameter and $S$ is rope tension, thus force on a micro-segment can be seen as constant.
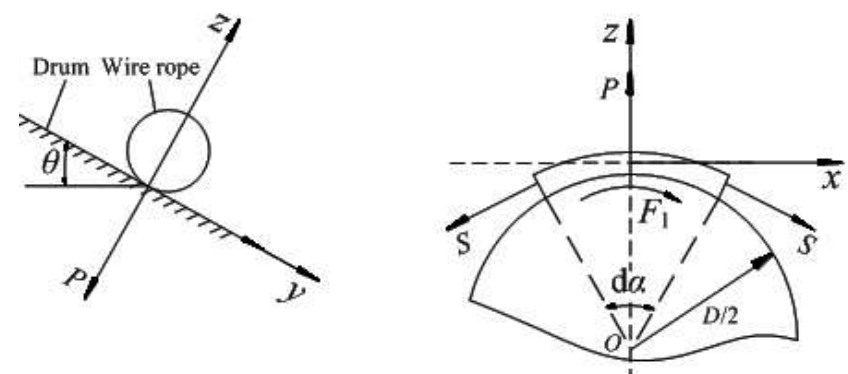

Fig. 2. Force analysis of contact modél.

Take unit length of the drum as research object, which subjects to normal load $P$ and traction friction force $F_{1}$ on the contact surface by wire rope

$$
\begin{gathered}
P=2 S \sin \theta / D \\
F_{1}=\sqrt{2} \mu S \sin \theta / D
\end{gathered}
$$

Rope wrapping is symmetric with respect to the drum axis, and belongs to line contact, thus, for independent effect of each force, contact model could be simplified as a two dimensional plane strain model between elastic cylinder and the plane.

\section{CONTACT STRESS ANALYSIS}

In the static contact state, based on the Hertzian theory, under normal load $P$, half width $a$ and normal pressure $p(x)$ are given as [9]

$$
\begin{gathered}
a=\sqrt{4 P R / \pi E} \\
p(x)=p_{0} \sqrt{1-x^{2} / a^{2}}
\end{gathered}
$$

with $p_{0}$ being the maximum normal pressure, $p_{0}=2 P / \pi \alpha ; R$ the rope diameter and $E$ the combined Yong's modulus, $1 / E=1 / E_{1}+1 / E_{2}, E_{1}, E_{2}$ are Yong's modulus of wire rope and the drum.; $x$ is the longitudinal distance between the contact point and the contact center.

The elastic stress components due to normal load $P$ at general points $(x, z)$ can be computed with the McEwen equations [11].

$$
\left(\sigma_{x}\right)_{P}=-\frac{p_{0}}{a}\left[m\left(1+\frac{z^{2}+n^{2}}{m^{2}+n^{2}}\right)-2 z\right]
$$

$$
\begin{gathered}
\left(\sigma_{z}\right)_{P}=-\frac{p_{0}}{a} m\left(1-\frac{z^{2}+n^{2}}{m^{2}+n^{2}}\right) \\
\left(\tau_{x z}\right)_{P}=-\frac{p_{0}}{a} n\left(\frac{m^{2}-z^{2}}{m^{2}+n^{2}}\right)
\end{gathered}
$$

where, $m$ and $n$ are defined by [10]:

$$
\begin{aligned}
& m^{2}=\frac{1}{2}\left[\left(a^{2}-x^{2}+z^{2}+4 x^{2} z^{2}\right)^{\frac{1}{2}}+a^{2}-x^{2}+z^{2}\right] \\
& n^{2}=\frac{1}{2}\left[\left(a^{2}-x^{2}+z^{2}+4 x^{2} z^{2}\right)^{\frac{1}{2}}-a^{2}-x^{2}+z^{2}\right]
\end{aligned}
$$

As for traction friction force $F_{1}$, according to the Carter solution [11]:

$$
\left\{\begin{array}{l}
\frac{q}{q_{o}}=\sqrt{1-\left(\frac{x}{a}\right)^{2}}-\frac{c}{a} \sqrt{1-\left(\frac{x-d}{c}\right)^{2}} \quad-a \leq x \leq-a+2 c \\
\frac{q}{q_{o}}=\sqrt{1-\left(\frac{x}{a}\right)^{2}} \quad-a \leq x \leq-a+2 c \\
q_{0}=-\mu p_{o}
\end{array}\right.
$$

where $c$ represents the half width of adhesion band and $d=$ $a-c . c$ is determined by the contact load through the following relationship:

$$
\frac{d}{a}=1-\frac{c}{a}=1-\left(1-\frac{|Q|}{\mu P}\right)^{1 / 2}
$$
loads.

with $Q$ being the total traction force and $P$ the total normal

Under traction friction force $F_{1}$, analytical expressions for stress components are given as

$$
\begin{gathered}
\left(\sigma_{x}\right)_{F_{1}}=-\frac{q}{a}\left[n\left(2-\frac{z^{2}-m^{2}}{m^{2}+n^{2}}\right)-2 x\right] \\
\left(\sigma_{z}\right)_{F_{1}}=-\frac{q}{a} n\left(\frac{m^{2}-z^{2}}{m^{2}+n^{2}}\right) \\
\left(\tau_{x z}\right)_{F_{1}}=\frac{q}{a}\left[m\left(1+\frac{z^{2}+n^{2}}{m^{2}+n^{2}}\right)-2 z\right]
\end{gathered}
$$

When contact between two objects with different elastic properties, if the limiting friction coefficient is obviously less than 1 , the interaction between tangential force and normal pressure is usually negligible [13]. Therefore, the stress components can be linearly superposed, the resulting expressions are:

$$
\sigma_{x}=\left(\sigma_{x}\right)_{P}+\left(\sigma_{x}\right)_{F_{1}}=-\frac{p_{0}}{a}\left[m\left(1+\frac{z^{2}+n^{2}}{m^{2}+n^{2}}\right)-2 z\right]-
$$




$$
\begin{gathered}
-\frac{q}{a}\left[n\left(2-\frac{z^{2}-m^{2}}{m^{2}+n^{2}}\right)-2 x\right] \\
\sigma_{z}=\left(\sigma_{z}\right)_{P}+\left(\sigma_{z}\right)_{F_{1}}=-\frac{p_{0}}{a} m\left(1-\frac{z^{2}+n^{2}}{m^{2}+n^{2}}\right)- \\
-\frac{q}{a} n\left(\frac{m^{2}-z^{2}}{m^{2}+n^{2}}\right) \\
\tau_{x z}=\left(\tau_{x z}\right)_{P}+\left(\tau_{x z}\right)_{F_{1}}=-\frac{p_{0}}{a} n\left(\frac{m^{2}-z^{2}}{m^{2}+n^{2}}\right)+ \\
+\frac{q}{a}\left[m\left(1+\frac{z^{2}+n^{2}}{m^{2}+n^{2}}\right)-2 z\right]
\end{gathered}
$$

\section{NUMERICAL SIMULATION OF FRETTING FATIGUE}

We can see from different development modes of fretting fatigue crack, the early stage of development is mainly affected by local fatigue on contact surface, the maximum surface tensile stress affects the formation of surface crack.
However, when the crack exceeds a certain depth, the local fatigue effect disappears and the overall fatigue plays a major role, at this time, the maximum internal shear stress influences the growth of fatigue crack. Therefore, the maximum surface tensile stress and internal shear stress are the main reasons for fretting fatigue damage, study on the distribution and impact factors are of great importance to the rational design and scientific maintenance of the drum.

\section{MAXIMUM SURFACE TENSILE STRESS}

Friction coefficient depends on the actual working conditions and material properties, it determines the contact state between two contact bodies [14]. In the friction drive process of endless rope winch, in order to prevent axial movement between the drum and wire rope, increase of the friction coefficient. However, that would affect the surface tensile stress, thereby influence the formation of surface cracks.

Substitute depth $z=0$ into Eq.(16), the distribution curves of surface tensile stress $\sigma_{x}$ with depth $z$ for different $\mu$ of 0.1 , 0.3 and 0.5 are shown in Fig.3.

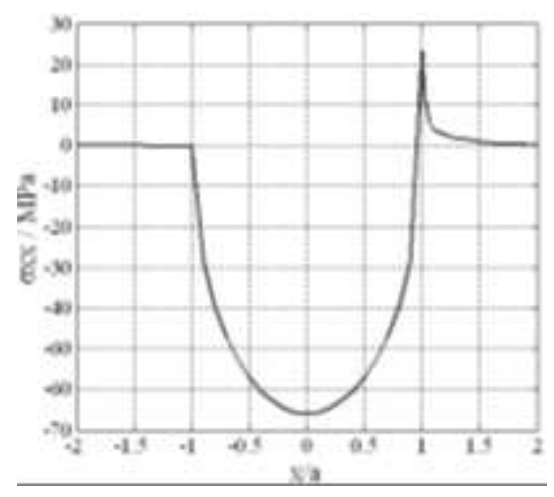

(a) $\mu=0.1$

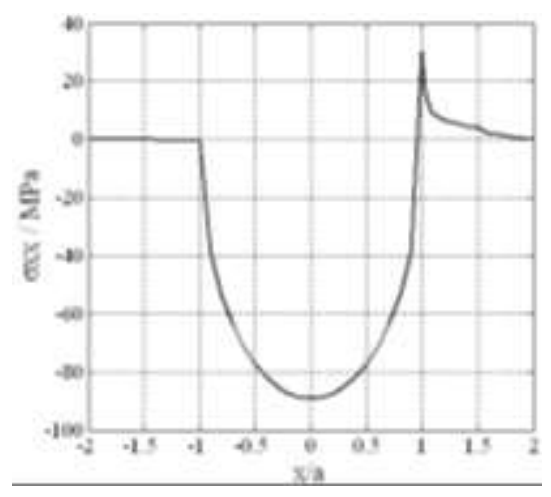

(b) $\mu=0.3$

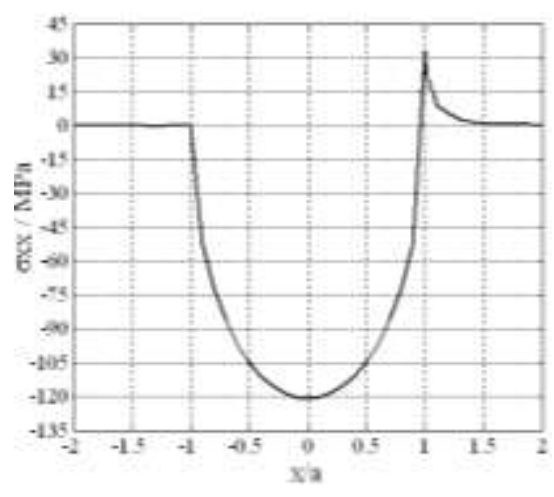

(c) $\mu=0.5$

Fig. 3. Relations between $\sigma \mathrm{x}$ and $\mathrm{z}$ with different $\mu$.

We find from Fig.3. that, the curves are symmetric with respect to the axis of $x=0$, with different friction coefficients, the distributions of surface stress remain unchanged, i.e. the maximum tensile stress occurs at $x=a$, and the maximum compressive stress emerges at $x=0$, only the value of maximum tensile stress changes with the friction coefficient.

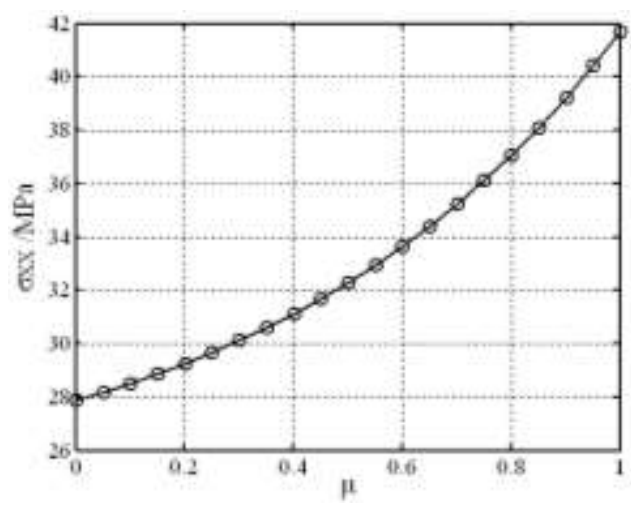

Fig. 4. Change curves between $\sigma_{\max }$ and $\mu$.
For further identification of the relations between the maximum tensile stress $\sigma_{\max }$ and friction coefficient $\mu$, with $\mu$ as abscissa and $\sigma_{\max }$ as ordinate, the change curves of $\sigma_{\max }$ with $\mu=0 \div 1$ are plotted in Fig. 4 .

In Fig.4, when $\mu=0, \sigma_{\max }$ is generated entirely by the normal load, thus its value is relatively small, only $27.76 \mathrm{MPa}$, as the increase of $\mu$, under the compound effect of the normal load and traction friction force, $\sigma_{\max }$ increases rapidly, the greater $\mu$ is, the effect of traction friction force becomes more obvious, when $\mu$ reaches 1 , the maximum tensile stress comes up to $41.68 \mathrm{MPa}$, which is very unfavorable for fatigue life of endless rope winch drum.

\section{SURROUNDING ANGLE}

Surrounding angle refers to the angle of wire rope wind onto the drum, by increasing the value of $\alpha$, it could obtain greater traction friction force, and improve the skid resistance of friction drive. Whereas, the maximum surface tensile stress would change with $\alpha$, while the curvature change of wire rope has a significant impact on its service life. 
Fig.5 and Fig.6 shows the variation curves of $\sigma_{\max }$ with $\alpha=0 \div 360^{\circ}$ for different friction coefficients $\mu$ and drum diameters $D$.

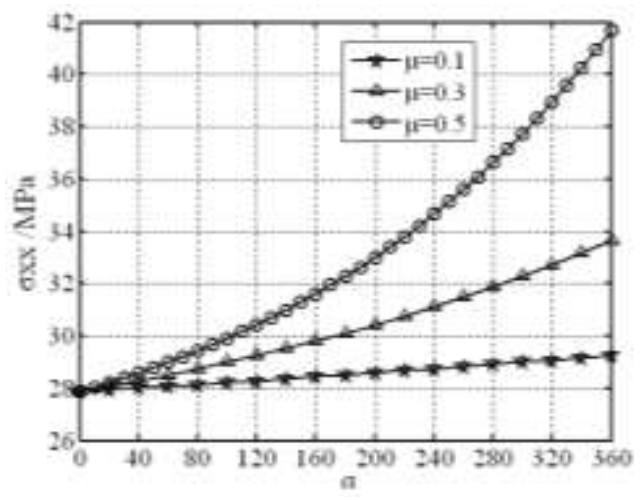

Fig. 5. Variation curves between $\sigma_{\max }$ and $\alpha$ For different $\mu$.

We find that $\sigma_{\max }$ is proportional to $\alpha$, and with the increase of $\mu$, the impact trend turns from linear relationship to exponential relationship, but for different $D$, the impact trends are similar, all close to linear relationship.

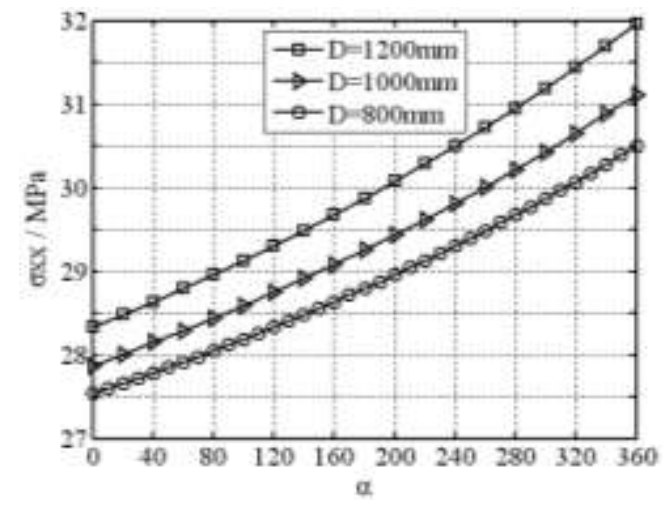

Fig. 6. Variation curves between $\sigma_{\max }$ and $\alpha$ for different $D$.

\section{COEFFICIENT OF CYLINDER GENERATRIX}

The cylinder generatrix is a straight line, the origin of coordinates is at the contact point, axis direction of the drum as $x$-coordinate direction, radial direction for $y$-coordinate direction, and the established coordinate system is shown in Fig.7.

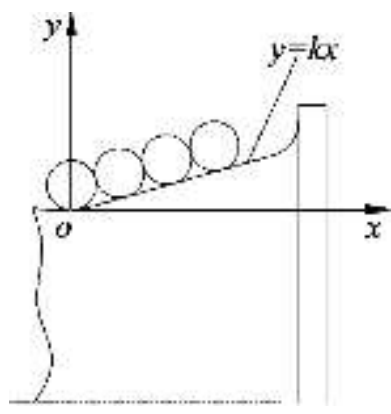

Fig. 7. Coordinate system for cylinder generatrix.

In this coordinate system, the equation for cylinder generatrix is:

$$
y=k x
$$

where, $k$ is the coefficient of cylinder generatrix, $k=\tan (\theta)$.

Fig.8. shows the relations between $\sigma_{\max }$ and $k$ with different $\mu: 0.1,0.3$ and 0.5 .

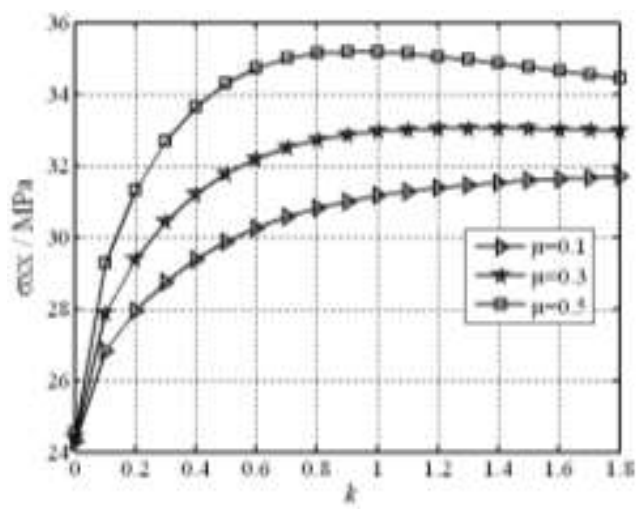

Fig. 8. Relations between $\sigma_{\max }$ and $k$ with different $\mu$.

We find that the influence trends are similar among different $\mu, \sigma_{\max }$ increases with the value of $k$, at first, the change amplitude is large, when $k$ reaches close to 1 , the increasing trend becomes gentle, while the increase of inclination angle would lead to larger extrusion pressure between wire ropes, and is prone to wear and occlusion, which adversely affect the friction drive.

\section{MAXIMUM INTERNAL SHEAR STRESS}

The contact fracture propagation is carried out under stress in type II, the maximum internal shear stress is a major source of stress in type II [15 17], Thus, analysis of its distribution is of importance to the research on propagation characteristics of contact fracture.

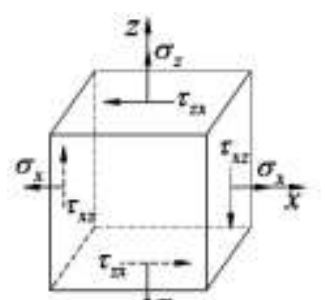

$(a)^{1 \sigma_{x}}$

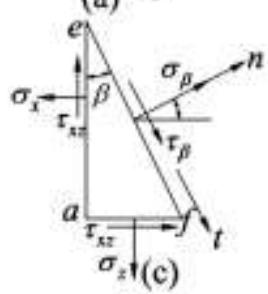

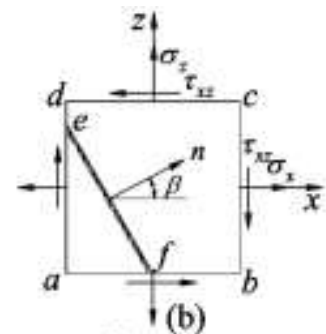

(b)

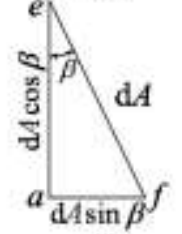

(d)
Fig. 9. Stress analysis of the unit

In the contact stress analysis, for independent effect of each force, the model was simplified as plane strain problem, stresses in certain directions were neglected. Force model of the internal contact zone was seen as two dimensional stress state, stresses on other sections were determined by stresses on 
a certain section, thus attained the principal stress and plane [18]. Fig.9. is stress analysis of the unit, on the surface, $\sigma_{x}, \sigma_{z}$, and $\tau_{x z}$ are all known.

Stresses on inclined section ef are expressed by normal stress $\sigma_{\beta}$ and shear stress $\tau_{\beta}, \mathrm{d} A$ represents area of ef, thus, areas of $a f$ and $a e$ are $\mathrm{d} A \sin \beta$ and $\mathrm{d} A \cos \beta$. Project forces on aef to outward normal $n$ and tangent $t$. According to analytical method for two-directional stress in mechanics of materials, we obtain:

$$
\left\{\begin{array}{l}
\sigma_{\beta}=\frac{\sigma_{x}+\sigma_{z}}{2}+\frac{\sigma_{x}-\sigma_{z}}{2} \cos 2 \beta-\tau_{x z} \sin 2 \beta \\
\tau_{\beta}=\frac{\sigma_{x}-\sigma_{z}}{2} \sin 2 \beta+\tau_{x z} \cos 2 \beta
\end{array}\right.
$$

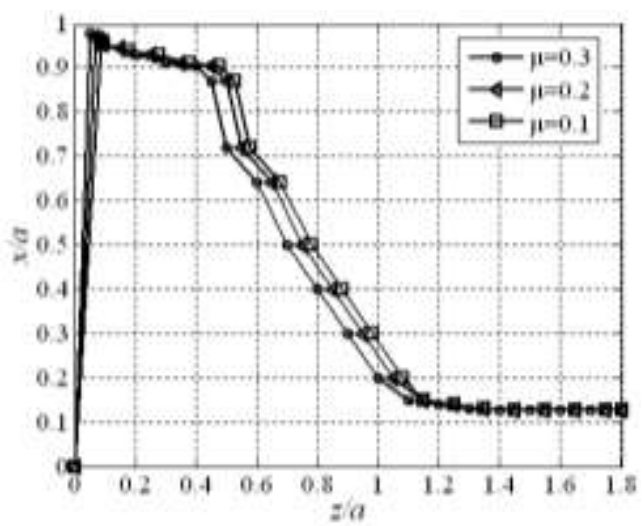

(a) Position-depth variation curves

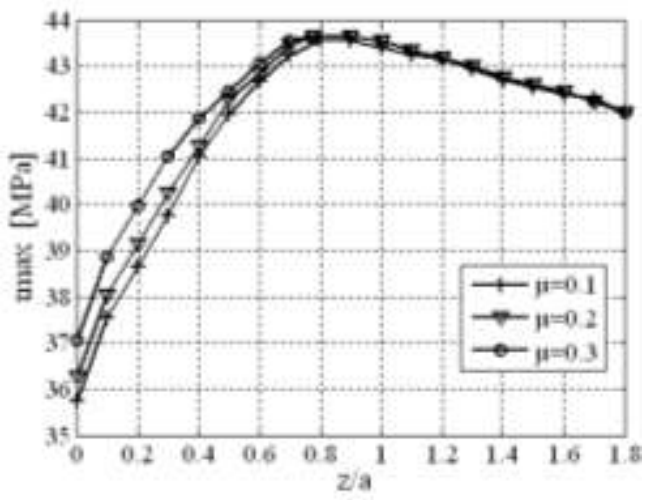

(b) Value-depth variation curves

Fig. 10. Variation curves between $\tau_{\max }$ and $z$ with different $\mu$

In above formulas, the normal stress $\sigma_{\beta}$ and shear stress $\tau_{\beta}$ change with the angle $\beta$, that means $\sigma_{\beta}$ and $\tau_{\beta}$ are functions of $\beta$, through derivation with respect to $\beta$ and make the derivative be 0 , the maximum and minimum shear stresses are expressed as

$$
\left.\begin{array}{l}
\tau_{\max } \\
\tau_{\min }
\end{array}\right\}= \pm \sqrt{\left(\frac{\sigma_{x}-\sigma_{z}}{2}\right)^{2}+\tau_{x z}^{2}}
$$

To probe the influence of friction coefficient $\mu$ on the maximum shear stress $\tau_{\max }$, according to the previous parameters, variation curves between $\tau_{\max }$ and depth $z$ with different $\mu$ : 0.1, 0.2 and 0.3 are shown in Fig. 10.

It can be seen from Fig.9, the occurrence position of $\tau_{\max }$ first quickly moves to the vicinity of $x=a$ with the increase of $z$, then slowly moves to $x=0$, when $z=1.4 a$, it is already close to $x=0.1 a$, its value also shows the trend of first increases and then deceases, it reaches the maximum value close to $z=0.8 a$.

Meanwhile, with the increase of $\mu, \tau_{\max }$ occurs closer to $x=a$, and the depth is of slight shallower, the value of $\tau_{\max }$ also shows an increase, but the change amplitude is very small, therefore, effect of friction coefficient is not obvious. Overall, in the transmission process, if the rope tension and traction friction force remain unchanged, the friction coefficient has little effect on maximum internal shear stress.

\section{CONCLUSIONS}

Based on the basic theories of microtribology and contact mechanics, the main reason for fretting fatigue of endless rope winch drum was analyzed. Then, numerical simulation study was carried out on the distribution and influencing factors of the maximum surface tensile stress and internal shear stress. The conclusions are obtained as follows:

1. Regardless of changes in the friction coefficient, the distribution of surface stress remains unchanged (i.e. the maximum tensile stress occurs at $x=a$ and the maximum compressive stress emerges at $x=0$ ). Along with the increase of friction coefficient, the value of maximum tensile stress becomes larger and the effect of traction friction force is more obvious.

2. Effect of the surrounding angle on maximum surface tensile stress increases with the friction coefficient, but for different drum diameters, the impact trends are all close to linear relationship.

3. The maximum surface tensile stress increases with the coefficient of cylinder generatrix. However, once its value is close to 1 , the increasing trend gradually becomes gentle.

4. In the transmission process, if the rope tension and traction friction force remain unchanged, the friction coefficient has little effect on maximum internal shear stress.

\section{Reference}

[1] L.V. Fedorova, Yu.S. Ivanova, M.V. Voronina, "Improvement of Threaded Joint Reliability by Means of Electromechanical Processing," Zapiski Gornogo instituta, Vol. 226, pp. 456-461, 2017.

[2] V.V. Nosov, "Control of Inhomogeneous Materials Strength by Method of Acoustic Emission," Zapiski Gornogo instituta, Vol. 226, pp. 4694792017.

[3] H.J. Jiang, Auxiliary transportation equipment in coal mine. Xuzhou: China University of Mining and Technology Press, 2008.

[4] Z.R. Zhou, "On fretting wear and fretting fatigue," China Mechanical Engineering, Vol. 11, pp. 1146-1150 2000.

[5] E.M. Eden, W.N. Rose, F.L. Cunningham, "The endurance of metals," Proceedings of the Institution of Mechanical Engineers, Vol. 4, pp. 839974, 1911.

[6] R.D. Mindlin, "Compliance of elastic bodies in contact," ASME Journal of Applied Mechanics, Vol. 16, pp. 259-268, 1949

[7] Z.R. Zhou, L. Vincent, Fretting wear. Beijing: Science Press, 2002.

[8] D.A. Hi11s, "Mechanics of fretting fatigue," Wear, Vol. 175, pp. 103107, 1994.

[9] D. Nowell, D.A. Hills, "Crack initiation criteria in fretting fatigue," Wear, Vol. 136, pp. 329-343, 1990. 
[10] W.G. Xue, "Shape and performance research on working mechanism of endless rope winch," Xuzhou: China University of Mining and Technology, 2006

[11] E. McEwen, "Stresses in elastic cylinders in contact along a generatrix," Philosophical Magazine, Vol. 40, pp. 454-457, 1949.

[12] F.W. Carter, "On the action of a locomotive driving whee," Proceedings of the Royal Society, Vol. 112, pp. 151-157, 1926.

[13] K.L. Johnson, Contact mechanics, London: Press Syndicate of the University of Cambridge, 1985.

[14] Y. Hong, "Contact stress analysis and fatigue life research on rotarysupport component of lager-scale rotary kiln under rolling contact," Changsha: Central South University, 2007.
[15] Y. Murakami, N.S. Nemat, "Growth and stability of interacting surface flaws of arbitrary shape," Engineering Fracture Mechanics, 17, 193$210,1983$.

[16] N. Yamashita, T. Mura, "Contact fatigue crack initiation under repeated oblique force,” Wear, Vol. 91, pp. 235-250, 1983.

[17] J.B. Sha, X.T. Jing, B.Z. Lou, Initiation and growth behavior of contact fatigue cracks. Acta Metallurgica Sinica, Vol. 31, pp. 422-430, 1995.

[18] H.W. Liu, Mechanics of material. Beijin: Higher Education Press, 2004. 